\title{
TIC para la determinación de los parámetros operacionales de humedales construidos diseñados para el tratamiento de aguas contaminadas por nitratos
}

\section{Resumen}

En España, debido a las prácticas agrícolas, el $13 \%$ del territorio ha sido declarado vulnerable a la contaminación del agua por nitratos. Para mitigar los impactos ambientales generados por el nitrógeno, se ha construido un sistema híbrido de humedales artificiales gracias al proyecto europeo LIFE REAGRITECH. El prototipo ha sido pensado como un sistema compacto, modular y móvil, automatizado y operado de forma remota. La monitorización del sistema producirá conocimientos valiosos para la Administración Pública, los agricultores, investigadores y universidades, y proporcionará información útil para mejorar la toma de decisiones con el fin de preservar los recursos hídricos y el medio ambiente. El proyecto REAGRITECH tiene grandes perspectivas de futuro en el campo de la agricultura en Europa y en Colombia.

Palabras clave: TIC, automatización y control, humedales construidos, escorrentía agrícola, contaminación por nitratos.

\footnotetext{
* Cátedra UNESCO de Sostenibilidad, Universitat Politècnica de Catalunya-BarcelonaTech, Terrassa, España, angel.gallegos.davalos@outlook.com

** Cátedra UNESCO de Sostenibilidad, UPC, lorena.aguilar@upc.edu

*** DASSA Technologies, Terrassa, España, i.campos@dassa.es

**** Grupo TYPSA, Barcelona, España, pcaro@typsa.es

***** Grupo TYPSA, Barcelona, España, ssahuquillo@typsa.es

****** LEITAT Technological Center, Terrassa, España, cperez@leitat.org

******** Department of Bioscience, University of Aarhus, Arhus C., Dinamarca, carlos.arias@bios.au.dk

Tecnológico de Antioquia, Coordinador UNESCOSOST Colombia, jorgemontoya@tdea.edu.co

Cátedra UNESCO de Sostenibilidad, UPC, jordi.morato@upc.edu
} 


\title{
ICTs for determining operational parameters of engineered wetlands designed for the treatment of nitrate-polluted water
}

\begin{abstract}
Due to the agricultural practices in Spain, 13\% of the entire territory has been declared as vulnerable to nitrate-polluted water. In order to mitigate the environmental impact caused by nitrogen, a hybrid system of artificial wetlands has been engineered thanks to the European project known as LIFE REAGRITECH. The prototype has been thought as a compact, modular, and portable system, automated and operated in a remote manner. Monitoring of the system will result in valuable knowledge for public management, farmers, researchers, and universities, and it will provide useful information intended to improve the decision-making process as a way of preserving water resources and environment. Project REAGRITECH offers significant future perspectives in the field of agriculture in Europe and in Colombia as well.
\end{abstract}

Key words: ICT; automation and control; engineered wetlands; agricultural overflow; nitrate pollution. 


\section{INTRODUCCIÓN}

En las últimas décadas las prácticas agrícolas han incrementado su producción a través de la optimización de los sistemas de riego y la adición a los cultivos de fertilizantes nitrogenados, fungicidas y pesticidas. Estas prácticas han ocasionado impactos directos sobre el recurso hídrico, como la contaminación de acuíferos y cuerpos de agua y, por lo tanto, una mayor explotación del recurso.

En la UE, entre el 50 y $80 \%$ del nitrógeno y el 30 y $80 \%$ del fósforo presentes en el agua se debe a las actividades agrícolas [1]. En España, un $80 \%$ de las aguas subterráneas tiene concentraciones superiores a los $25 \mathrm{mgL}^{-1} \mathrm{de} \mathrm{NO}_{3}$ [2] y un $13 \%$ del territorio ha sido declarado como zona vulnerable por nitratos, donde se presentan concentraciones superiores a los $50 \mathrm{mgL}^{-1}[3]$.

Actualmente se dispone de tratamientos que permiten reutilizar directamente el agua en el riego agrícola, utilizando tecnologías naturales de tratamiento, como los humedales construidos $(\mathrm{CW})$, donde la remoción de nitrógeno total varía entre $40 \mathrm{y}$ $55 \%$, para un rango de carga entre 250 y $630 \mathrm{gr} \mathrm{m}^{2} \mathrm{y}^{-1}$, dependiendo de su tipología y la carga de entrada [4].

La automatización de estos sistemas permitiría seguramente aumentar la eficiencia y productividad de los sistemas de explotación [5], y al mismo tiempo preservar el medio ambiente. De acuerdo con Calera [6], una de las características esenciales de un sistema eficaz y duradero de gestión colectiva del recurso hídrico es la existencia de una estructura adecuada de vigilancia y control de las extracciones, por lo que la incorporación de las tecnologías de la información a la agricultura permite obtener información útil en tiempo real para una mejor toma decisiones.

Por otra parte, las unidades de producción agrícola presentan necesidades y requerimientos en el área de la tecnología de la información y las comunicaciones (TIC) que pueden apoyar los procesos técnicos con mayor eficiencia, confiabilidad y seguridad [7]. El uso de TIC para la operación y control del humedal construido del proyecto REAGRITECH coincide con la necesidad del acceso del mundo rural al uso de estas nuevas tecnologías, en condiciones semejantes a las del medio urbano [8].

\section{MATERIALES Y MÉTODOS}

El proyecto LIFE REAGRITECH "Regeneration and reuse of runoff and drainage water in agricultural plots by combined natural water treatment systems" utiliza los $\mathrm{CW}$ como una tecnología natural de tratamiento para aguas contaminadas por nitratos, con la finalidad de disminuir los impactos que tienen en el medio ambiente y, al mismo tiempo, para reducir las extracciones y el consumo del recurso hídrico destinado a 
las actividades agrícolas, por medio de su reutilización en el riego de la parcela. Los objetivos específicos del proyecto son:

- Reutilizar el agua de escorrentía y de infiltración para el riego de parcelas.

- Adaptar los sistemas de tratamiento y las tecnologías apropiadas a las prácticas agrícolas.

- Mejorar la calidad del agua superficial y subsuperficial declarada como vulnerable por nitratos.

- Reducir los costos de tratamiento de agua.

El proyecto busca contribuir sustancialmente en el desarrollo de los objetivos de sostenibilidad enmarcados dentro de las políticas de la Unión Europea y ser un ejemplo en la gestión sostenible de los recursos hídricos.

El prototipo desarrollado para el proyecto REAGRITECH permite determinar valores específicos para las constantes cinéticas $\mathrm{k}$, en aguas contaminadas por nitratos procedentes de las prácticas agrícolas, mediante un diseño experimental de tratamiento de distintas cargas contaminantes (DBO y NT) que permitan determinar constantes cinéticas en un período específico de tiempo.

REAGRITECH pretende demostrar un método para reutilizar los recursos hídricos a escala de parcela, con el fin de optimizarlos para su mejor uso en el ecosistema y, en consecuencia, lograr una cuenca fluvial sostenible e integrada, mediante la mejora de las características químicas del agua reutilizada y, por tanto, del entorno natural que les rodea.

La operación y recolección de información sobre el funcionamiento del prototipo se realiza a través de un sistema de domótica adaptado al proyecto; su flexibilidad permite controlar y conocer el estado, en tiempo real, de cada uno de los componentes dados de alta en la plataforma de operación.

\subsection{Diseño del sistema}

Se ha diseñado un CW híbrido (piloto demostrativo) para determinar la constante $\mathrm{k}$ específica para al agua contaminada por nitratos provenientes de la agricultura. El dimensionamiento se ha realizado mediante el método $\mathrm{PkC}^{*}$, donde $\mathrm{k}$ es una constante cinética que depende del tipo de contaminante, y de la temperatura en la cual se llevan a cabo los procesos de remoción, y donde $\mathrm{C}^{*}$ representa la concentración de fondo del humedal [9].

La configuración del sistema de tratamiento presenta un humedal vertical (VFCW) de $5.5 \mathrm{~m}^{2} \mathrm{y}$ dos humedales horizontales (HFCW) de $4,10 \mathrm{~m}^{2}$ funcionando en paralelo. 
En uno de los HFCW se ha utilizado como medio granular corcho triturado de 12 $\mathrm{mm}$, y grava de $32 \mathrm{~mm}$, en el otro, para poder comparar las eficiencias de remoción que presentan.

El diseño contempla la circulación del agua a través de los distintos humedales; así se puede iniciar el tratamiento por el humedal vertical y pasar posteriormente por los horizontales, y viceversa, así como recircularla en el mismo humedal en la etapa que se considere necesario, con la finalidad de aumentar la remoción de contaminantes.

\subsection{Construcción}

El piloto demostrativo se ha realizado con un innovador proceso constructivo basado en el uso de contenedores marítimos, como se puede ver en las figuras 1 y 2 . Se trata de un prototipo compacto, modular y móvil que no causa impacto en el terreno: compacto, por la recirculación del efluente entre los distintos humedales construidos, alcanza remociones de contaminantes más altas en un área menor; modular, por incrementar la superficie de tratamiento, y agrega más contenedores y modifica algunos elementos destinados a su operación (bombas, electroválvulas, etc.), y móvil, porque puede ser trasportado a otra ubicación. El prototipo se ha cubierto mediante una cubierta (figura 3) y una pared vegetal con placas de corcho como base (figura 4), para protegerlo de los efectos de temperaturas extremas, las cuáles podrían afectar el funcionamiento de los componentes electrónicos. La vegetación, además de mantener el confort térmico dentro de los contenedores marítimos, le permite al prototipo adaptarse al paisaje agrícola de la zona (figura 5).

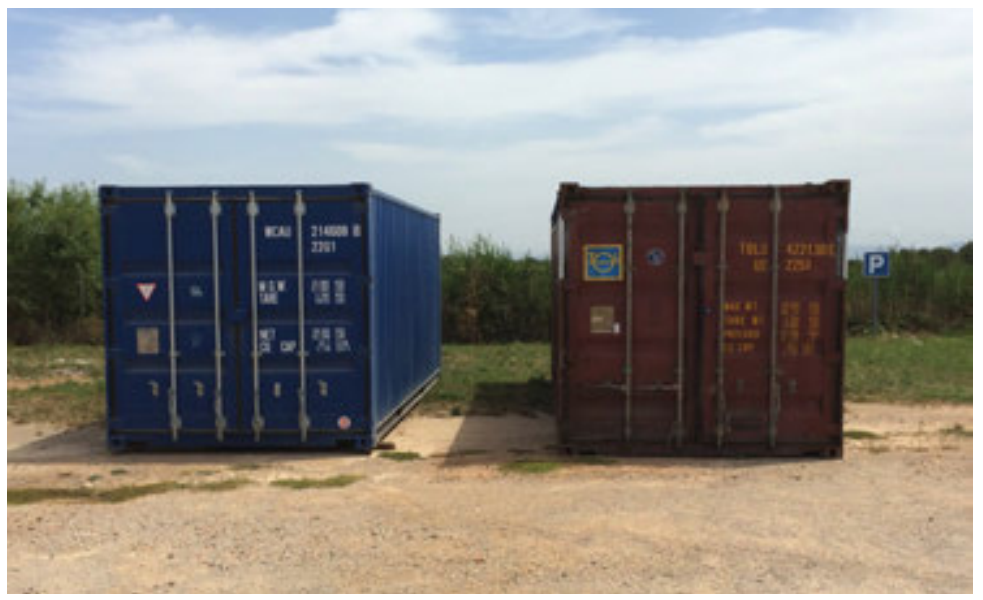

Figura 1. El uso de contenedores marítimos para la construcción del sistema de tratamiento permite su trasladado a las parcelas donde se requiera la descontaminación del agua.

Fuente. [10] 


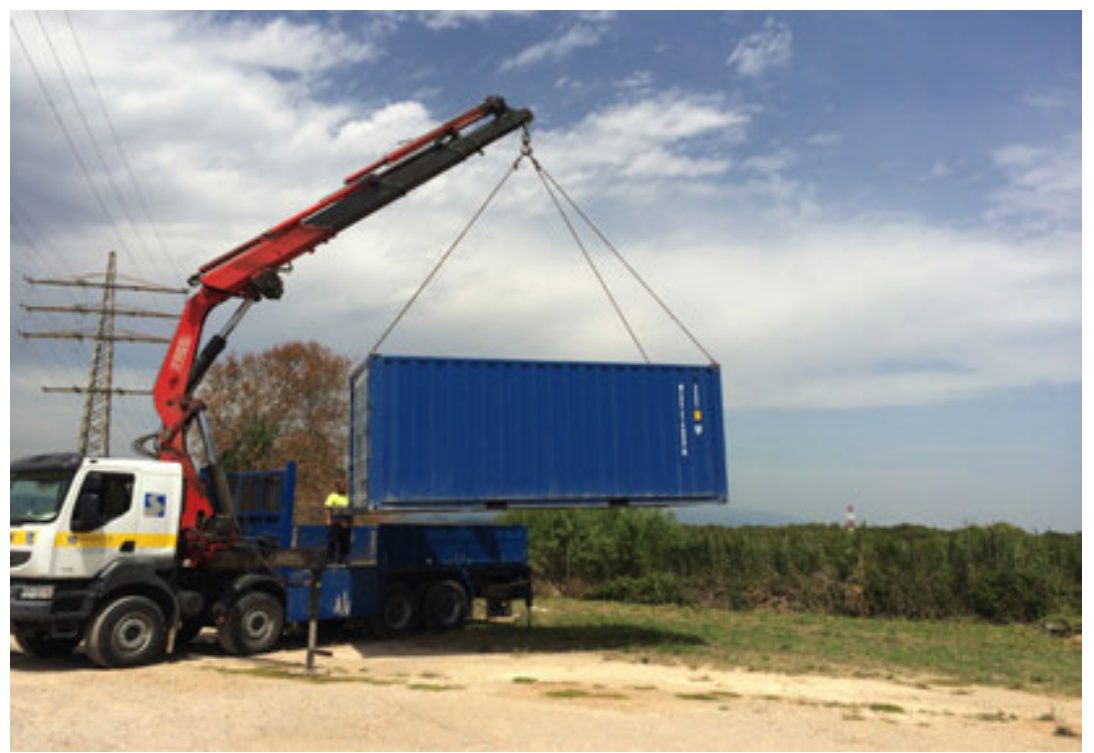

Figura 2. El piloto puede ser transportado por medio de un camión grúa o con pluma a las distintas ubicaciones donde sea requerido.

Fuente. [10]

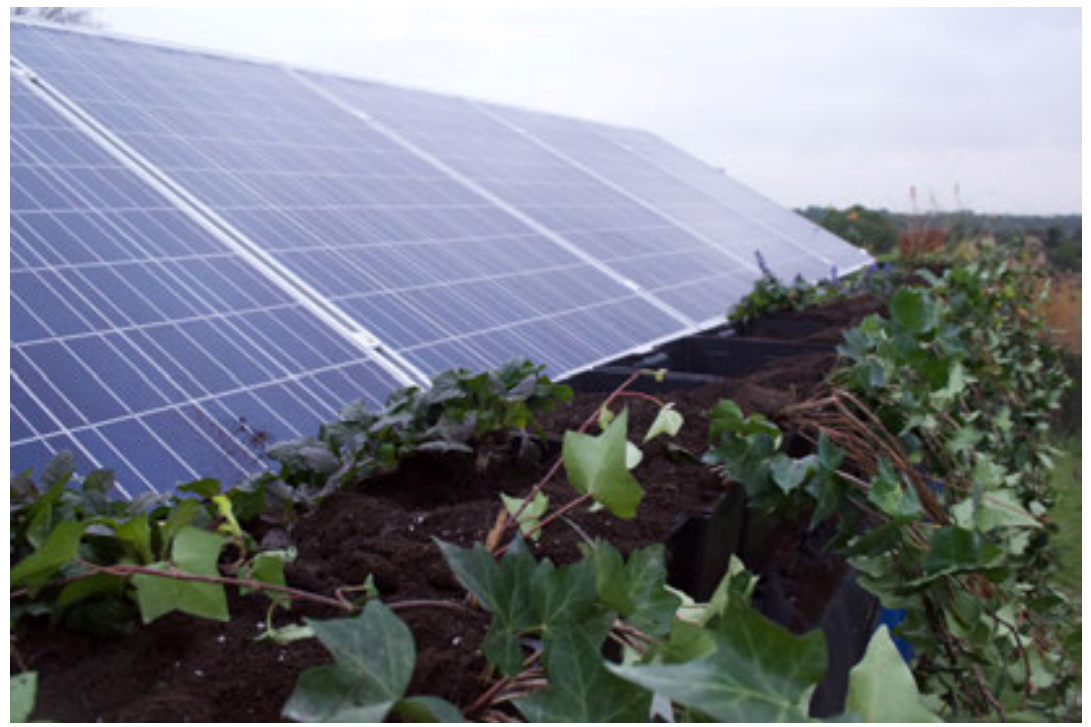

Figura 3. El sustrato de la cubierta vegetal se encuentra contenido

dentro de un sistema modular de plástico reciclado, lo cual le permite también el ser desmontado a la hora de transportarse el prototipo. 


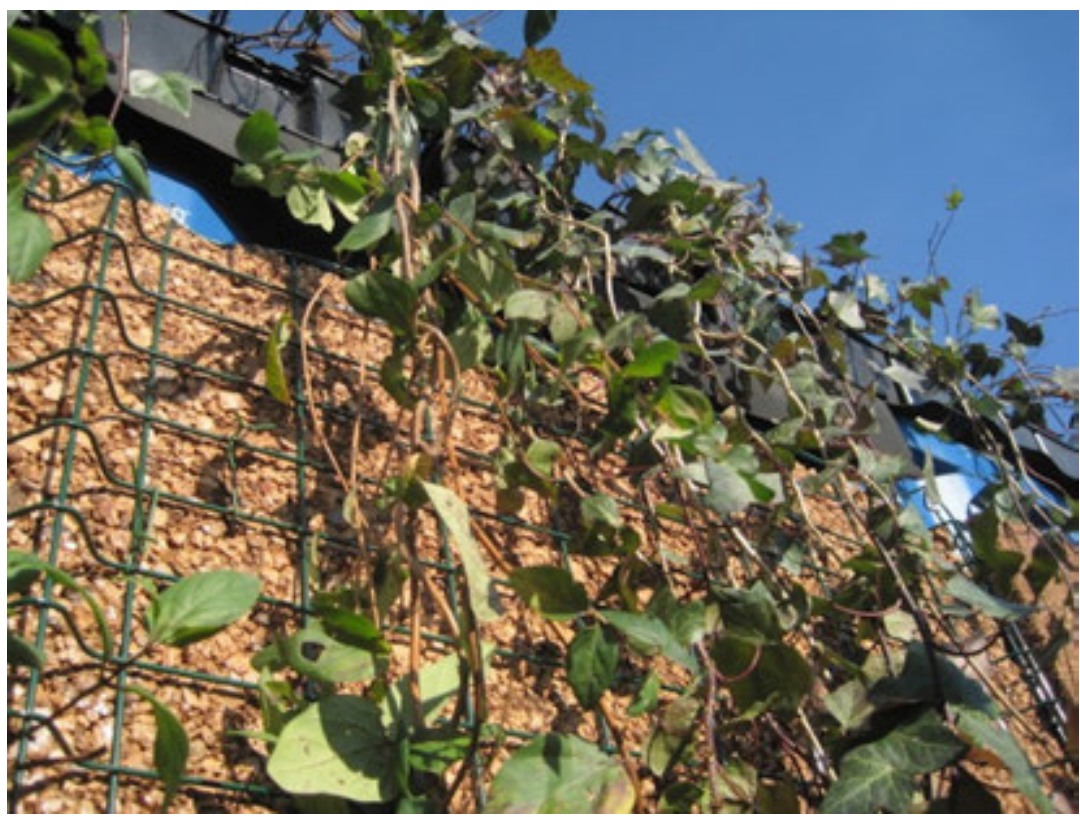

Figura 4. La pared vegetal, en combinación con el corcho, permite tener temperaturas en verano, dentro del contenedor, cercanas a los $29^{\circ} \mathrm{C}$.

Fuente. [11]

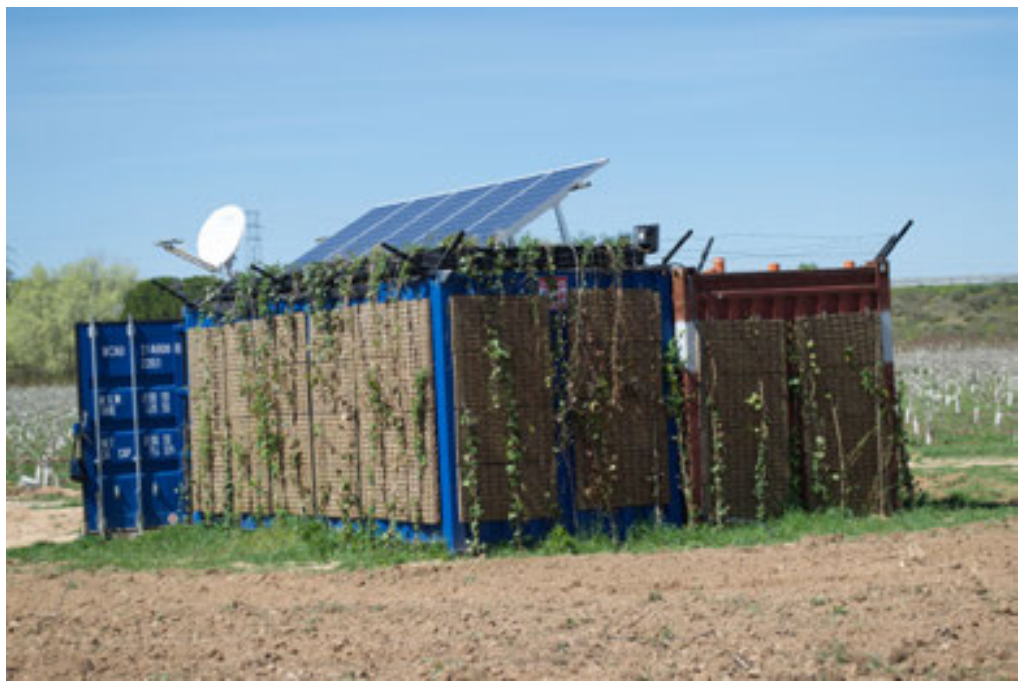

Figura 5. La vegetación de la cubierta y la pared vegetal contemplan especies nativas que no generen un impacto visual en el entorno.

Fuente. [11] 


\subsection{Operación y monitorización}

El afluente del humedal se bombea a partir de dos piezómetros construidos en la parcela agrícola, que captan el agua subsuperficial en la zona. El tratamiento primario se realiza en un tanque de sedimentación de donde puede conducirse a cualquiera de los humedales (HFCWs o VFCW), ya que cada uno tiene su propio depósito de bombeo y de recogida. Los elementos de la domótica instalada permiten tener un sistema flexible, donde se puede decidir cuál es el ciclo que se debe hacer de acuerdo con las características del agua: tratar el afluente por el VFCW y posteriormente por los HFCW, o viceversa, así como recircularlo, según se muestra en la figura 6. Adicionalmente, el sistema permite la operación del prototipo de forma automática y permite crear y modificar las reglas de funcionamiento de cada componente electrónico del sistema (bombas de flujo, caudalímetros, electroválvulas, sensores de temperatura y de nivel, etc.).

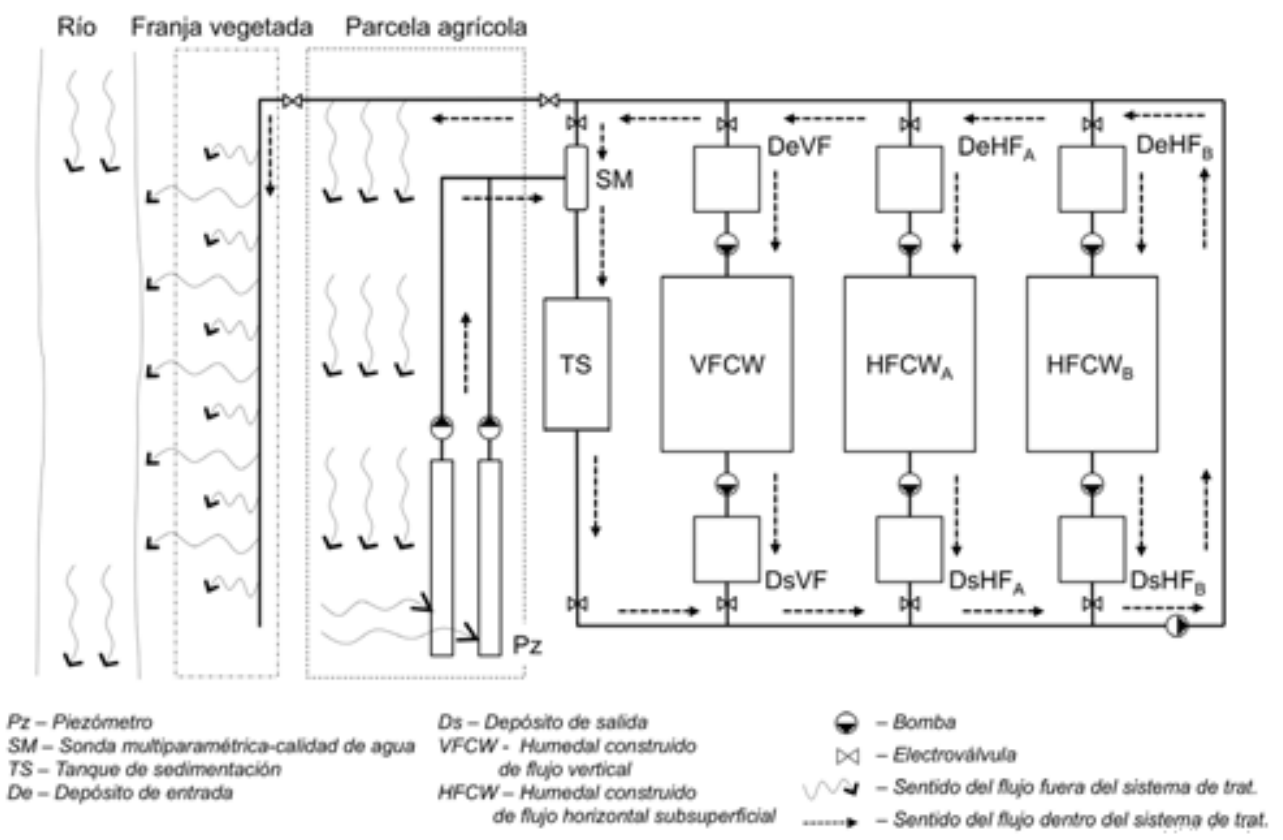

Figura 6. Esquema de diseño del piloto demostrativo de tratamiento mediante humedales construidos.

Fuente. [12] 
Un sistema base web permite la monitorización a distancia y ajustar los parámetros de operación según las necesidades del proyecto (cantidad y características del afluente y efluente, condiciones climatológicas, etc.). La plataforma web, presentada en la figura 7, divide el sistema por zonas, diferenciando cada uno de los elementos que lo componen: elementos de entrada del efluente, humedal vertical, humedal horizontal A y $\mathrm{B}$, zonas comunes.

\section{DHS - Domotic Home System}

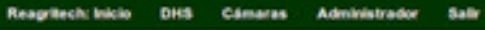

Sesión iniciada éxitosamente

Zonas - Vista Global

2. Todo

- Vntrada Aqua

4 Humedal Horizontal A

- 7 Deposito Entrada Hum Hor A

* Deposito Salida Hum Hor A

7. Humedal Horizontal B

* : Deposito Entrada Hum Hor B

- Deposito Salida Hum Hor B

0

7. Humedal Vertical

- Deposito Entrada Hum Vertical

- Deposito Salida Hum Vertical

Z Zonas Comunes

Copyright 2013 DaSSA Technologies S.L. Todos los derechos reservados

Telefono: +34937834754 - Contact

Figura 7. Plataforma web con visualización del sistema por zonas.

Fuente. [13]

A su vez, en cada una de las zonas mostradas en la figura 8, puede visualizarse cada elemento vinculado a ella, como son los sensores de nivel, bombas, electroválvulas, contadores de consumo, filtros y caudalímetros de las figuras 9 y 10. Las figuras 11 a 12 muestran todos estos elementos, los cuales están controlados por un kit de automatización que consta de computadora, ruteador, módulos de control, potencia y distintos sensores. 


\section{d)}

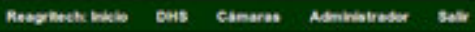

\section{Lista de Sensores - Zona Humedal Horizontal A}

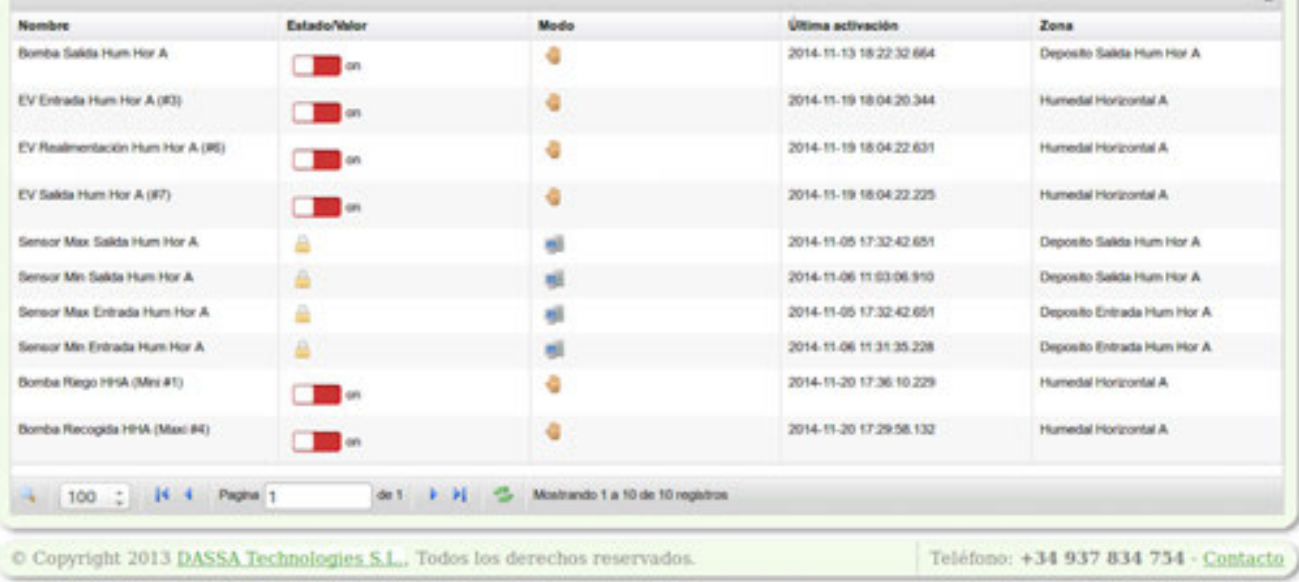

Figura 8. Página web con detalles de la zona del humedal Horizontal A, con el detalle de cada elemento en el prototipo.

Fuente. [13] 


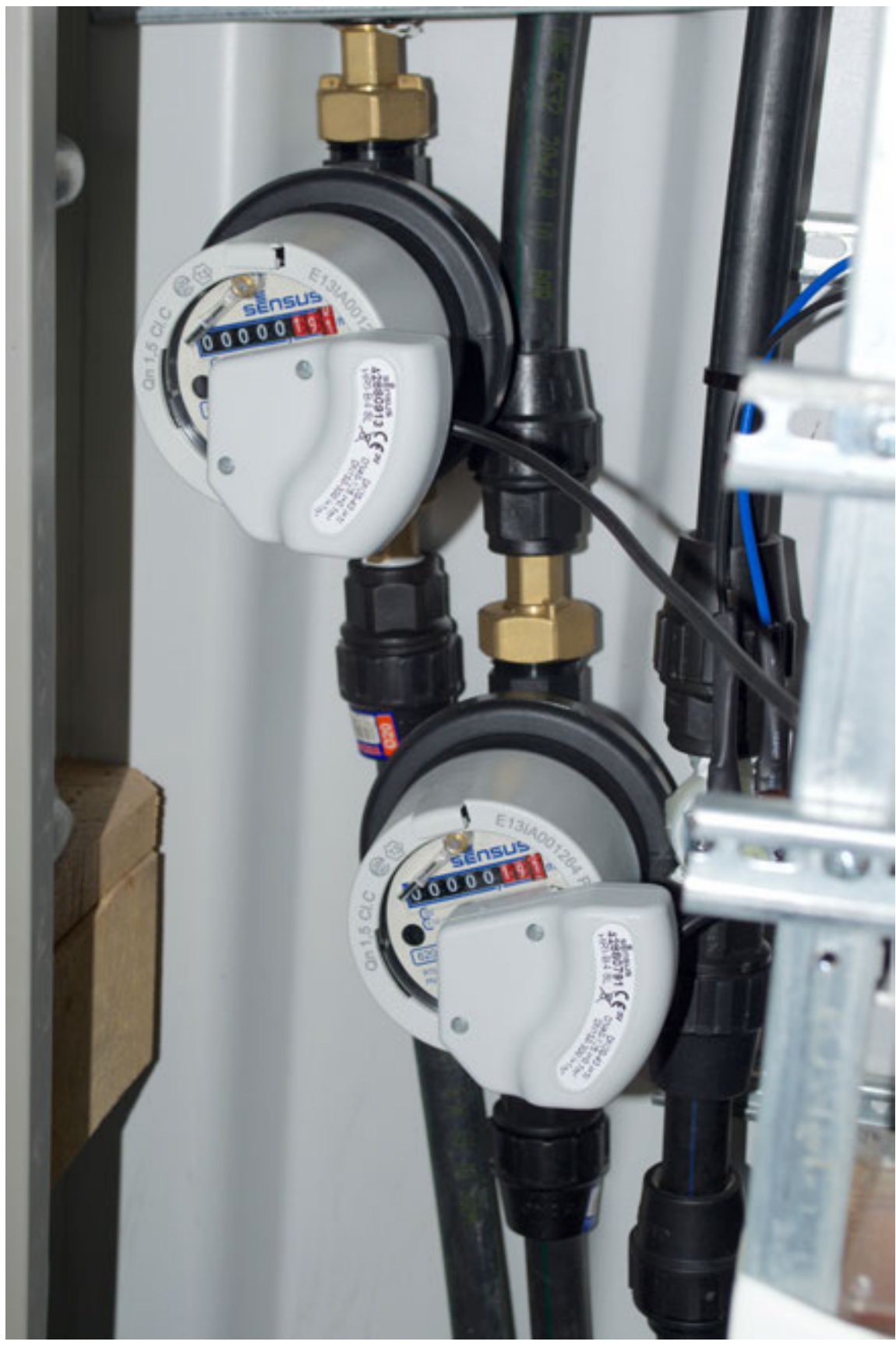

Figura 9. Contador de agua para medir el caudal de entrada a cada humedal del piloto Fuente. [11] 


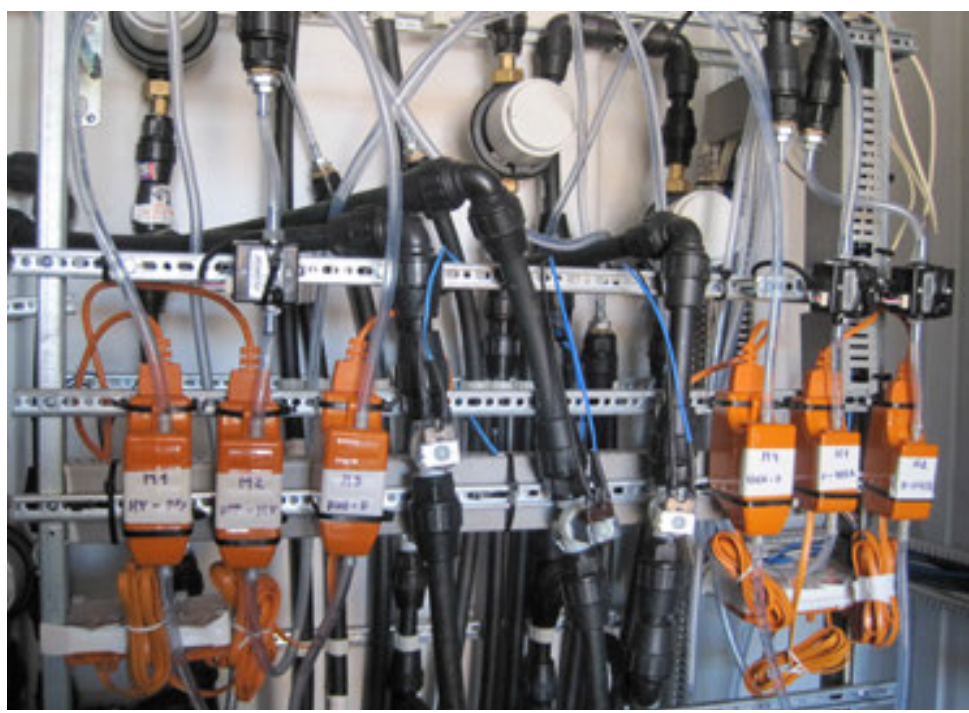

Figura 10. Elementos del cuadro de control hidráulico del piloto. bombas, sensores de nivel, electroválvulas.

Fuente. [11]

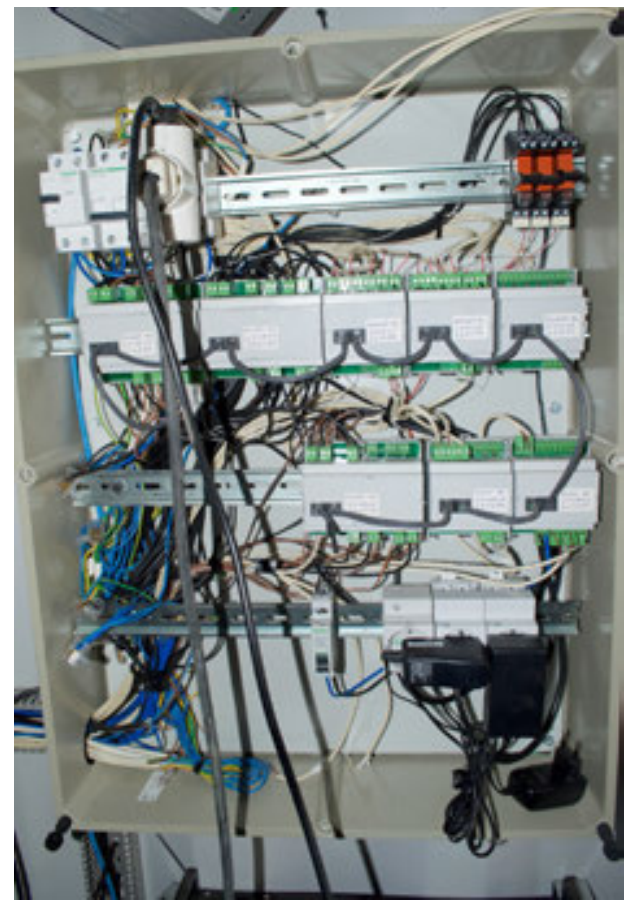

Figura 11. Módulos de control y fuerza diseñados específicamente para el proyecto.

Fuente. [11] 
Este sistema adaptado de domótica, a diferencia de otros sistemas descentralizados existentes, interactúa con el usuario sin que este note su presencia, hace más confortable, segura y eficiente la monitorización del piloto y aporta, además, toda la información en bases de datos de cada uno de los elementos dados de alta en el sistema (figuras 13 y 14). El sistema de control es capaz de tomar decisiones en situaciones complejas, ofreciendo al usuario algo más que una simple automatización o control remoto de los dispositivos.

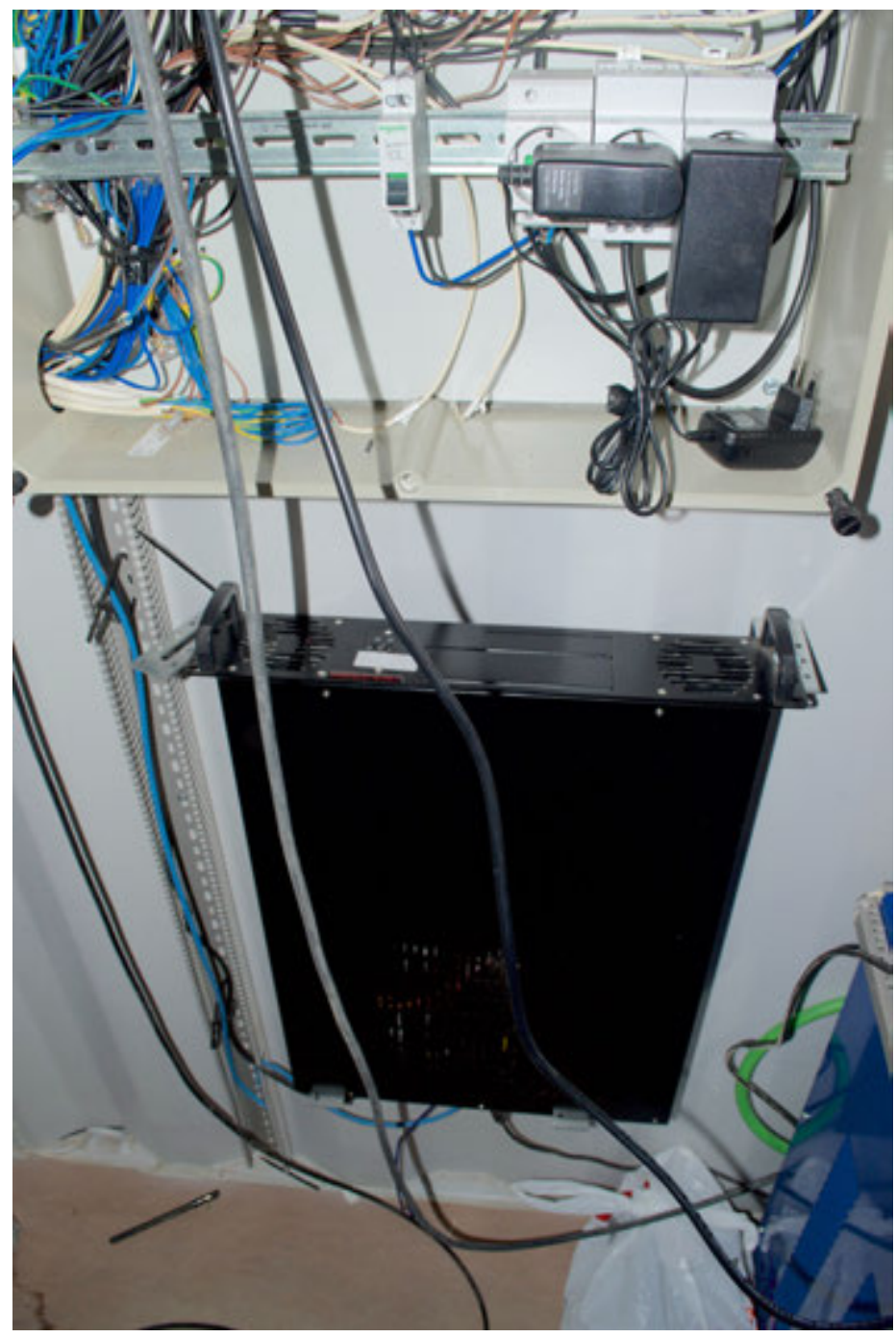

Figura 12. El CPU encargado de controlar la automatización está fijado en la pared del contenedor.

Fuente. [11] 


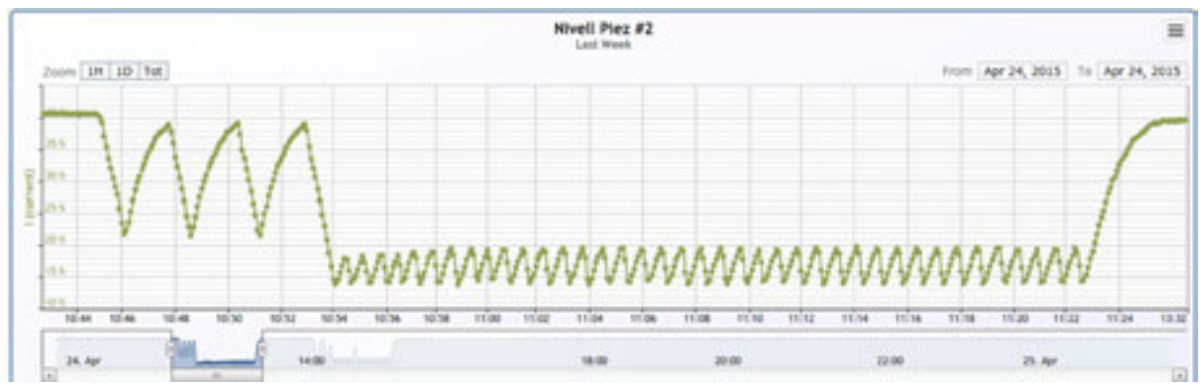

Figura 13. La plataforma permite la realización de gráficas de operación en tiempo real de cada uno de los elementos del sistema para facilitar la toma de decisiones.

Fuente. [13]

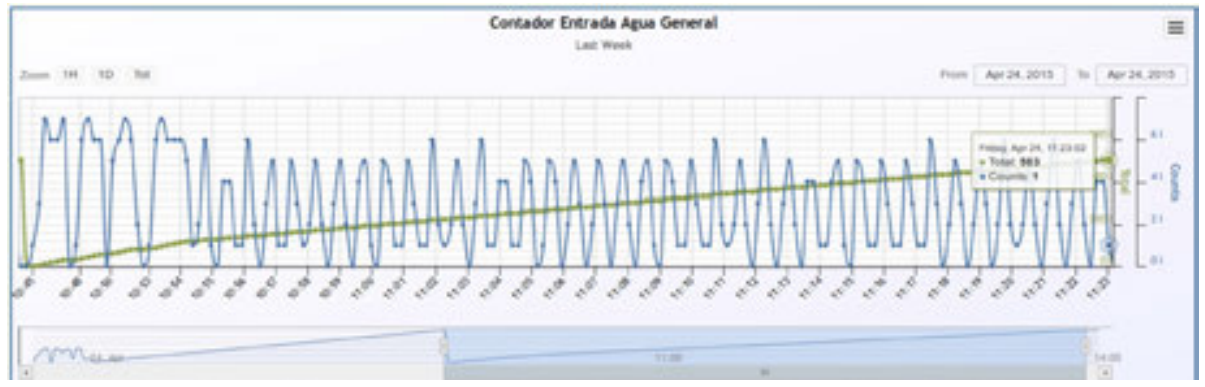

Figura 14. El sistema aporta la información de funcionamiento de cada uno de los elementos dados de alta en su base de datos.

Fuente. [13]

\section{RESULTADOS}

El objetivo del diseño es obtener valores de la constante cinética k para agua contaminada por nitratos procedentes de actividades agrícolas que permita obtener efluentes por debajo de 5 mgL-1 de NH4-N y 10 mgL-1 de NO3-N, establecidas por Ayers [10], para ser reutilizados en el riego de parcelas.

Se están realizando campañas de muestreo mensuales de tres días consecutivos en 7 meses (julio, agosto, septiembre y noviembre de 2015, y en febrero y mayo de 2016), para validar la eficiencia del primer prototipo. Las diferencias entre el medio granular de los humedales horizontales permitirán comparar la eficiencia de remoción del sistema entre un sustrato tradicional, como la grava, y un subproducto de otras actividades, como el corcho.

La adaptación de la domótica para ser utilizada en el proyecto ha brindado una solución integral para la operación del prototipo REAGRITECH, dando al usuario final un nivel superior de confort, seguridad y ahorro energético. La automatización 
de los ciclos y procedimientos de depuración, así como la rapidez para medir datos confiables en tiempo real, ha permitido un ahorro significativo en los recursos técnicos, humanos, económicos y del tiempo disponible para cumplir los objetivos del proyecto.

\section{DISCUSIÓN}

La escasez hídrica y la contaminación de los acuíferos plantea retos tecnológicos, económicos y sociales cada vez más relevantes. Este proyecto permitirá encontrar parámetros de diseño específicos para optimizar el tratamiento de aguas contaminadas con nitratos provenientes de actividades agrícolas por medio de humedales construidos y, de esta manera, ser reutilizadas en la agricultura con la finalidad de reducir el consumo de agua de esta actividad, mitigar los impactos que generan los nitratos en el medio ambiente, y valorar el uso y eficiencia del corcho como medio filtrante en los humedales, figuras 15 y 16 .

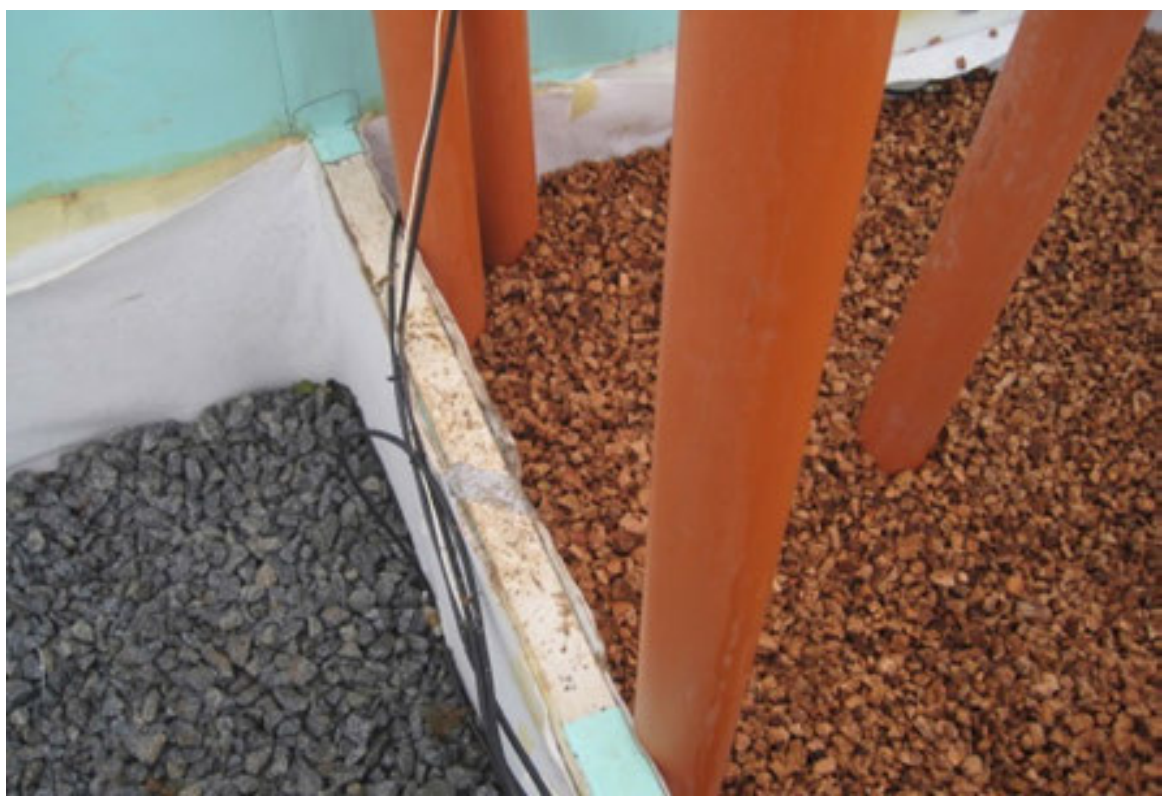

Figura 15. Materiales granulares (grava y corcho) utilizados en los humedales híbridos. Fuente. [15] 


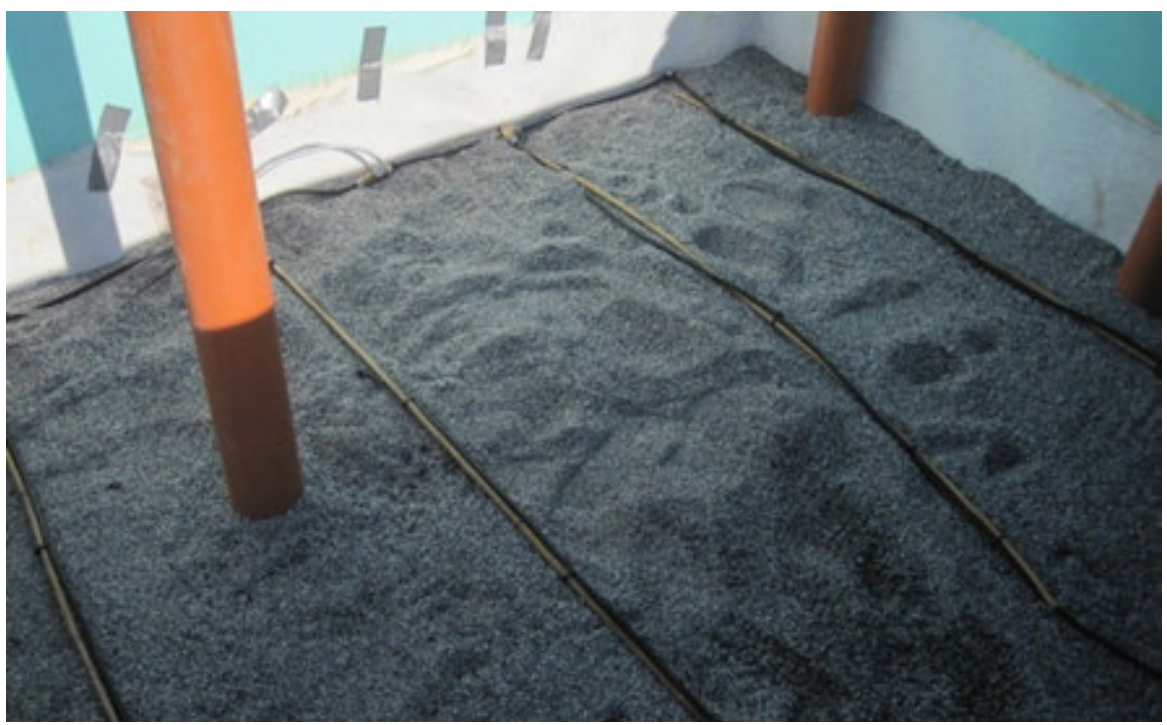

Figura 16. Sobre el material granular se coloca el sistema de distribución que alimenta al humedal con el agua contaminada.

Fuente. [15]

\section{CONCLUSIONES}

Las herramientas TIC permiten contar con un nivel superior de interactividad, capaz de controlar las instalaciones de forma realmente autónoma y cubrir la necesidad de obtención de información sobre las variables necesarias para mejorar el diseño y operación de los $\mathrm{CW}$, así como para la recopilación y conformación de una base de datos del sistema que permitan tomar decisiones sobre su operación.

La plataforma web del proyecto es una excelente herramienta que pone a la disposición de los usuarios finales, así como de entidades de la Administración Pública e investigadores, el conocimiento necesario para la toma de decisiones encaminadas a la optimización del recurso hídrico y al cuidado del medio ambiente.

El proyecto, además de poseer un carácter fuertemente demostrativo, es representativo y reproducible en todo el territorio europeo y ha sido pensado para ayudar en la implementación de las políticas de la Unión Europea en los ámbitos de la sostenibilidad y mejora del medio ambiente.

\section{AGRADECIMIENTOS}

Al Programa LIFE+ de la Dirección General de Medio Ambiente de la Comisión Europea que ha subvencionado el proyecto REAGRITECH LIFE11 ENV/ES/579. 


\section{REFERENCIAS}

[1] K. Isermann, "Share of agriculture in nitrogen and phosphorus emissions into the surface waters of Western Europe against the background of their eutrophication", Fertil. Res 26, 253-269,1990.

[2] European Environment Agency Present concentration of nitrate in groundwater bodies in European countries, 2007.

[3] L. Fernández, "Enseñanza de las Ciencias de la Tierra", (15.3), 257-265, 2007.

[4] J. Vymazal, "Removal of nutrients in various types of constructed wetlands", Sci. Total Environ. 380, 48-65, 2007.

[5] A. Pérez, M. Milla, M. Mesa, "Impacto de las tecnologías de la información y la comunicación en la agricultura", Cultivos tropicales, 27(1), 11-17, 2006.

[6] A. Calera, I. Campos, "El uso de la teledetección en la gestión del agua en la agricultura de regadío. Incorporación de la teledetección a la gestión del agua en la agricultura. Monográfico riegos del alto Aragón. Enero 2010, 13-15, 2010.

[7] J. Chaparro, B. Pérez, “Agro-Tic's una Visión tecnológica integrada e interactiva de información para el desarrollo agrícola en Venezuela", 11th Latin American and Caribbean Conference for Engineering and Technology, Cancún, México, August 14-16, 2013.

[8] J. Morillo, M. Bellido, E. Gordillo, "La agricultura y las nuevas tecnologías", La agricultura y las ganaderías extremeñas en 2005, Cap. 19, 383-401, 2005.

[9] R. Kadlec, S. Wallace, "Treatment wetlands," 2nd edition, Taylor \& Francis Group, 2009.

[10] Á. Gallegos. Archivo fotográfico LIFE REAGRITECH ENV/ES/579 [Fotografía]. Cátedra UNESCO de Sostenibilidad de la Universitat Politècnica de Catalunya-BarcelonaTech, 2014.

[11] J. Morató. Archivo fotográfico LIFE REAGRITECH ENV/ES/579 [Fotografía]. Cátedra UNESCO de Sostenibilidad de la Universitat Politècnica de Catalunya-BarcelonaTech, 2014.

[12] Á. Gallegos. Esquema de funcionamiento de los humedales construidos para el proyecto REAGRITECH [Figura]. Cátedra UNESCO de Sostenibilidad de la Universitat Politècnica de Catalunya-BarcelonaTech, 2014.

[13] DASSA Technologies. Plataforma web del proyecto REAGRITECH [Figura], 2014.

[14] R. Ayers, D. West Cot, "La calidad del agua en la agricultura", Estudio FAO, Serie Riego y drenaje. $\mathrm{N}^{\circ}$ 29. Rev. Ed. FAO. 85, 1987.

[15] L. Aguilar. Archivo fotográfico LIFE REAGRITECH ENV/ES/579 [Fotografía]. Cátedra UNESCO de Sostenibilidad de la Universitat Politècnica de Catalunya-BarcelonaTech, 2014. 
